\title{
Examine different weed management techniques in sugarcane (Saccharum officinarum L.)
}

\author{
Qamarddin Jogi ${ }^{1 *}$, Ghulam Ali Hajano ${ }^{1}$, Muhammad Nawaz Kandharo ${ }^{1}$, \\ Ahmed Naqi Shah ${ }^{1}$, Aijaz Ahmed Soomro ${ }^{1}$, Zulfiqar Ali Abbasi ${ }^{1}$, \\ Mahmooda Burioro ${ }^{1}$, Shahla Karim Baloch ${ }^{2}$, Zameer Ahmed Kalwar ${ }^{1}$, \\ Naveed Ahmed Abbasi ${ }^{1}$ and Khalid Hussain Banglani ${ }^{1}$ \\ 1. Department of Agronomy, Sindh Agriculture University, Tandojam-Pakistan \\ 2. Department of Biotechnology, Sindh Agriculture University, Tandojam-Pakistan \\ *Corresponding author's email: qdjogi@gmail.com
}

Citation

Qamarddin Jogi, Ghulam Ali Hajano, Muhammad Nawaz Kandharo, Ahmed Naqi Shah, Aijaz Ahmed Soomro, Zulfiqar Ali Abbasi, Mahmooda Burioro, Shahla Karim Baloch, Zameer Ahmed Kalwar, Naveed Ahmed Abbasi and Khalid Hussain Banglani. Examine different weed management techniques in sugarcane (Saccharum officinarum L.). Pure and Applied Biology. Vol. 8, Issue 1, pp151-159. http://dx.doi.org/10.19045/bspab.2018.700173

\begin{tabular}{llll}
\hline \hline Received: 04/08/2018 & Revised: 03/10/2018 & Accepted: 18/10/2018 & Online First: 25/10/2018 \\
\hline
\end{tabular}

\section{Abstract}

The study was carried out during the year 2016-17 to examine different weed management techniques in sugarcane (Saccharum officinarum L.). The experiment was laid out at Sugarcane Section, Agriculture Research Institute, Tandojam in a three replications RCBD with (8 $\mathrm{m} \times 3 \mathrm{~m}$ ) $24 \mathrm{~m}^{2}$ size of plot. In current investigation the study area was made up of four weed species were recorded which included Chaff-flower, Green amaranth, Creeping thistle and Lamb's quarters. The above record of the weed flora was maintained sowing of sugarcane. The sugarcane crop treated with hand hoeing thrice resulted in $94.27 \mathrm{~m}^{-2}$ weed density, $39.33 \%$ weed reduction, $192.00 \mathrm{~cm}$ cane length, $2.05 \mathrm{~cm}$ cane girth, 6.13 tillers stool $^{-1}, 14.45 \mathrm{~kg}$ weight of 10 canes, $58.13 \mathrm{t} \mathrm{ha}^{-1}$ cane yield, $20.33 \%$ brix and $10.17 \%$ sugar recovery. The crop treated with weedy check produced $155.45 \mathrm{~m}^{-2}$ weed density, $0.00 \%$ weed reduction, $161.67 \mathrm{~cm}$ cane length, $1.73 \mathrm{~cm}$ cane girth, 2.98 tillers stool ${ }^{-1}, 6.68 \mathrm{~kg}$ weight of 10 canes, $27.22 \mathrm{t} \mathrm{ha}^{-1}$ cane yield, $18.94 \%$ brix and $9.47 \%$ sugar recovery. It was concluded that the crop treated with Buctril M @ $3.75 \mathrm{~kg} \mathrm{ha}^{-1}$ resulted in highest cane height, girth, density and yield.

Keywords: Management; Sugarcane; Techniques; Weed

\section{Introduction}

Sugarcane (Sachharum officinarum L.) is cultivated for sugar production, while among by-products bagasse is used for chip board and hard board production and used as fuel in the factory as well. The molasses is used for manufacture of chocolate and menthol; while the use of factory wastes (press mud and spent wash) are useful to improve soil fertility [1, 2]. During 2015-16, the sugarcane crop stood at 1132 thousand hectares compared to last year's area of 1141 thousand hectares showing a decline of 0.8 percent. Sugarcane production for the year 2015 increased to 65.5 million tons from 62.8 million tons of last year's production 
showing an increase of 4.2 percent. The decline in area is due to disposal problem of cane and payment difficulties restricted acreage of sugarcane that shifted sugarcane area to other competitive crops. The increase in production is due to favorable weather condition [3]. The interspecies competition between the cane and the intercrops for nutrients and water can be minimized, if adequate irrigation is provided to both the crops. This can also prevent the risk of reduced can yield. Hence the longer values and small duration of sugarcane crop is mainly based on system of cropping that had enough potential for enhancing land utilization efficiency to save water for irrigation purpose and reduction in cost of production for making sugarcane crop sustainable. Northern areas of India various methods of cultivating and growing crops such as soybean, potato, common bean, wheat, rapeseed, linseed, peas and vegetables with inter spaces of sugarcane with collectively inter-crops having higher popularity in farmers.

[4].The weed control practices in the sugarcane plantations of the country are a combination of manual weeding and herbicide application [5]. In plant-crop the weeds are usually managed four times during the crop growth periods. These include hand weeding, pre emergence followed by post emergence herbicide application and hand weeding. However, competitive suppression of weeds can take a very different form with intercropping than in crop monocultures. Increasing the complexity of a cropping system 3 by inter planting species of differing growth forms, phonologies and physiologies can create different patterns of resource availability to weeds, especially light [6].

\section{Materials and methods}

Present experiment was conducted at Sugarcane Section, Agriculture Research Institute, Tandojam in a three replicated (RCBD) during the year 2016-17 having plot size of $8 \mathrm{~m} \mathrm{x} 3 \mathrm{~m}\left(24 \mathrm{~m}^{2}\right)$. A better quality seedbed was adopted during the land preparation procedures. The ridges made to place various sets of seed by the method of end to end. The seed setts were dipped in fungicidal solution before sowing to avoid incidence of any seed borne disease. The fertilizers ( $\mathrm{N}, \mathrm{P}$ and $\mathrm{K}$ ) were applied at the rates of 220,120 and $100 \mathrm{~kg} \mathrm{ha}^{-1}$ respectively. All $\mathrm{P}$ and $\mathrm{K}$ and $1 / 3^{\text {rd }}$ of $\mathrm{N}$ was applied at planting time and remaining $\mathrm{N}$ in two equal doses at first earthing $\left(3^{-1} / 2\right.$ months after planting) and $1^{-1} / 2$ month after first earthing, respectively. The following weed control treatments were tested.

\section{Observations to be recorded}

1. Weed flora of sugarcane

2. Weed density $\left(\mathrm{m}^{-2}\right)$

3. Weed reduction $(\%)$

4. Cane length $(\mathrm{cm})$

5. Cane girth $(\mathrm{cm})$

6. Tillers stool ${ }^{-1}$

7. Weight of 10 canes $(\mathrm{kg})$

8. Cane yield $\left(\mathrm{t} \mathrm{ha}^{-1}\right)$

9. Brix (\%)

Sugar recovery $(\%)$

\section{Statistical analysis}

The data were subjected to statically analysis using Statics 8.1 computer software [7]. The difference among the treatment means were compared L.S.D test where necessary.

\section{Results}

Present investigation was performed in the year of 2016 for examining different weed management techniques in sugarcane (Saccharum officinarum L.). The experiment was laid out at Agriculture Research Institute, Sugar Section, Tandojam in 3 replications RCBD with (8 m x $3 \mathrm{~m}$ ) $24 \mathrm{~m}^{2}$ size of plot.

\section{Weed flora}

Weeds are an extra factor that sometimes modifies the plant stand drastically. In this study in the experimental plots comprised of three wheat varieties, six weed species were recorded which included Chaff-flower, 
Green amaranth, Creeping thistle and Lamb's quarters. The above record of the weed flora was maintained sowing of sugarcane (Table$1)$.

Table 1. Weed flora found the experimental field of sugarcane

\begin{tabular}{|c|c|c|c|}
\hline English Name & Local Name & Botanical Name & Family \\
\hline Chaff-flower & Sanskrit & $\begin{array}{c}\text { Achyranthes aspera } \\
\text { Linn }\end{array}$ & Amaranthaceae \\
\hline Green amaranth & Slender amaranth & $\begin{array}{c}\text { Amaranthus virdis } \\
\text { Linn. }\end{array}$ & Amaranthaceae \\
\hline Creeping thistle & Prickly thistle & $\begin{array}{c}\text { Cirsium arvense (L.) } \\
\text { scop. }\end{array}$ & Asteraceae \\
\hline Lamb's quarters & White goosefoot & $\begin{array}{c}\text { Chenopodium album } \\
\text { Linn. }\end{array}$ & Amaranthaceae \\
\hline
\end{tabular}

\section{Weed density $\left(\mathrm{m}^{-2}\right)$}

The sugarcane crop attained maximum weed density $\left(155.45 \mathrm{~m}^{-2}\right)$ when under weedy check, closely followed by $94.27 \mathrm{~m}^{-2}, 71.52$ $\mathrm{m}^{-2}$ and $53.94 \mathrm{~m}^{-2}$ weed density in plots receiving hand hoeing thrice, hand hoeing twice and hand hoeing once. A simultaneous reduction in weed density of the sugarcane crop i.e. $44.16 \mathrm{~m}^{-2}, 37.81 \mathrm{~m}^{-2}$ and $36.70 \mathrm{~m}^{-2}$ were recorded in plots receiving inter-row cultivation only, hand weeding once + interrow cultivation and Metribuzin + 2, 4-D @ $3.75 \mathrm{~kg} \mathrm{ha}^{-1}$ on cane rows + inter-row cultivation, respectively. However, the shortest weed density on average $\left(30.76 \mathrm{~m}^{-2}\right)$ was recorded in control plots, where crop treated with Buctril M @ $3.75 \mathrm{~kg} \mathrm{ha}^{-1}$ (Table 2).

Table 2. Weed density $\left(\mathrm{m}^{-2}\right)$ of sugarcane as influenced by various weeds treatments

\begin{tabular}{|c|c|c|c|c|}
\hline Treatment & R 1 & R II & R III & Mean \\
\hline TI =Buctril M @ 3.75 kg ha & -1 & 30.31 & 32.33 & $\mathbf{3 0 . 7 6 ~ G}$ \\
\hline $\begin{array}{c}\text { T2=Metribuzin + 2, 4-D @ 3.75 } \mathrm{kg} \mathrm{ha}^{-1} \text { on cane rows } \\
+ \text { inter-row cultivation }\end{array}$ & 36.63 & 38.12 & 35.64 & $\mathbf{3 6 . 7 0 ~ F}$ \\
\hline T3=Hand weeding once + inter-row cultivation & 38.43 & 36.56 & 38.44 & $\mathbf{3 7 . 8 1} \mathbf{~ F}$ \\
\hline T4=Inter-row cultivation only & 43.91 & 43.56 & 45.00 & $\mathbf{4 4 . 1 6} \mathbf{~}$ \\
\hline T5=Hand hoeing once & 51.62 & 53.33 & 56.86 & $\mathbf{5 3 . 9 4} \mathbf{D}$ \\
\hline T6=Hand hoeing twice & 69.20 & 72.66 & 72.71 & $\mathbf{7 1 . 5 2} \mathbf{C}$ \\
\hline T7=Hand hoeing thrice & 90.91 & 93.54 & 98.36 & $\mathbf{9 4 . 2 7} \mathbf{~ B}$ \\
\hline T8=Weedy check & 158.38 & 151.32 & 156.66 & $\mathbf{1 5 5 . 4 5 ~ A}$ \\
\hline
\end{tabular}

$\mathrm{SE} \pm=1.7500, \mathrm{LSD} 0.05=3.7534$

\section{Weed reduction $(\%)$}

The sugarcane crop attained maximum weed reduction $(80.20 \%)$ when crop treated with Buctril M@3.75 kg ha-1, closely followed by $76.37 \%, 75.68 \%$ and $71.59 \%$ weed reduction in plots receiving Metribuzin +2 , 4-D@3.75 kg ha-1 on cane rows + inter-row cultivation, Hand weeding once + inter-row cultivation and Inter-row cultivation only. A simultaneous decline in weed reduction of the sugarcane crop i.e. $65.29 \%, 53.96 \%$ and $39.33 \%$ were recorded in plots receiving Hand hoeing once, Hand hoeing twice and Hand hoeing thrice, respectively. However, the shortest weed reduction on average $(0.00 \%)$ was recorded in control plots, where crop treated with weedy check (Table 3 ). 
Table 3. Weed reduction (\%) of sugarcane as influenced by various weed treatment

\begin{tabular}{|c|c|c|c|c|}
\hline Treatment & R I & R II & R III & Mean \\
\hline T1 =Buctril M @ $3.75 \mathrm{~kg} \mathrm{ha}^{-1}$ & 81.29 & 79.97 & 79.36 & $80.20 \mathrm{~A}$ \\
\hline $\begin{array}{c}\mathrm{T} 2=\text { Metribuzin }+2,4-\mathrm{D} @ 3.75 \mathrm{~kg} \mathrm{ha}^{-1} \text { on cane rows }+ \text { inter- } \\
\text { row cultivation }\end{array}$ & 77.06 & 74.81 & 77.25 & 76.37 B \\
\hline T3=Hand weeding once + inter-row cultivation & 75.74 & 75.84 & 75.46 & $75.68 \mathrm{~B}$ \\
\hline T4=Inter-row cultivation only & 72.28 & 71.21 & 71.28 & $71.59 \mathrm{C}$ \\
\hline T5=Hand hoeing once & 67.41 & 64.76 & 63.70 & 65.29 D \\
\hline T6=Hand hoeing twice & 56.31 & 51.98 & 53.59 & $53.96 \mathrm{E}$ \\
\hline T7=Hand hoeing thrice & 42.60 & 38.18 & 37.21 & $39.33 \mathrm{~F}$ \\
\hline T8=Weedy check & 0.00 & 0.00 & 0.00 & $0.00 \mathrm{G}$ \\
\hline
\end{tabular}

$\mathrm{SE} \pm=0.9760, \mathrm{LSD} 0.05=2.0933$

\section{Cane length (cm)}

The maximum length of cane in sugarcane crop was observed $(268.3 \mathrm{~cm})$ when crop treated with Buctril M @ $3.75 \mathrm{~kg} \mathrm{ha}^{-1}$, closely followed by $260.0 \mathrm{~cm}, 256.3 \mathrm{~cm}$ and 253.6 $\mathrm{cm}$ cane length in plots receiving hand weeding once + inter-row cultivation, Metribuzin + 2, 4-D @ $3.75 \mathrm{~kg} \mathrm{ha}^{-1}$ on cane rows + inter-row cultivation and hand hoeing twice. A simultaneous decline in cane length of the sugarcane crop i.e. $247.0 \mathrm{~cm}, 242.6 \mathrm{~cm}$ and $192.0 \mathrm{~cm}$ were recorded in plots receiving inter-row cultivation only, hand hoeing once and hand hoeing thrice, respectively. However, the shortest cane length on average $(161.6 \mathrm{~cm})$ was recorded @ weedy check, where crop treated with weedy check (Table-4).

Table 4. Cane length cm of sugarcane as influenced by various weed treatments

\begin{tabular}{|c|c|c|c|c|}
\hline Treatment & R I & R II & R III & Mean \\
\hline TI =Buctril M @ 3.75 $\mathrm{kg} \mathrm{ha}^{-1}$ & 270.00 & 275.00 & 260.00 & $\mathbf{2 6 8 . 3}$ \\
\hline $\begin{array}{c}\text { T2=Metribuzin + 2, 4-D @ 3.75 kg ha }{ }^{-1} \text { on cane rows + } \\
\text { inter-row cultivation }\end{array}$ & 261.00 & 250.00 & 258.00 & $\mathbf{2 5 6 . 3 ~ B}$ \\
\hline $\begin{array}{c}\text { T3=Hand weeding once + inter-row cultivation } \\
\text { T4=Inter-row cultivation only }\end{array}$ & 275.00 & 251.00 & 254.00 & $\mathbf{2 6 0 . 0 ~ B}$ \\
\hline T5=Hand hoeing once & 251.00 & 242.00 & 248.00 & $\mathbf{2 4 7 . 0 ~ C D ~}$ \\
\hline T6=Hand hoeing twice & 248.00 & 242.00 & 238.00 & $\mathbf{2 4 2 . 6 ~ D}$ \\
\hline T7=Hand hoeing thrice & 256.00 & 250.00 & 255.00 & $\mathbf{2 5 3 . 6 ~ B C}$ \\
\hline T8=Weedy check & 195.00 & 191.00 & 190.00 & $\mathbf{1 9 2 . 0}$ \\
\hline
\end{tabular}

$\mathrm{SE} \pm=4.2496, \mathrm{LSD} 0.05=9.1146$

\section{Cane girth $(\mathrm{cm})$}

The sugarcane crop attained maximum cane girth $(3.08 \mathrm{~cm})$ when crop treated with Buctril M @ $3.75 \mathrm{~kg} \mathrm{ha}^{-1}$, closely followed by $3.03 \mathrm{~cm}, 2.56 \mathrm{~cm}$ and $2.37 \mathrm{~cm}$ cane girth in plots receiving Metribuzin +2, 4-D @ 3.75 $\mathrm{kg} \mathrm{ha}{ }^{-1}$ on the rows of cane+ inter row cultivation. Hand weeding once+inter- row cultivation and inter row cultivation only. A simultaneous decline in cane girth of the sugarcane crop i.e. $2.28 \mathrm{~cm}, 2.17 \mathrm{~cm}$ and 2.05 $\mathrm{cm}$ were recorded in plots receiving hand hoeing once, hand hoeing twice and hand hoeing thrice, respectively. However, the shortest cane girth on average $(1.73 \mathrm{~cm})$ was recorded @ weedy check, where crop treated with weedy check (Table 5). 
Table 5. Cane girth $\mathrm{cm}$ of sugarcane as influenced by various weed treatments

\begin{tabular}{|c|c|c|c|c|}
\hline Treatment & R I & R II & R III & Mean \\
\hline TI =Buctril M @ 3.75 $\mathrm{kg} \mathrm{ha}^{-1}$ & 3.15 & 3.06 & 3.03 & $\mathbf{3 . 0 8} \mathbf{~ A}$ \\
\hline $\begin{array}{c}\text { T2=Metribuzin + 2, 4-D @ 3.75 kg ha }{ }^{-1} \text { on cane rows + inter-row } \\
\text { cultivation }\end{array}$ & 3.06 & 3.03 & 3.00 & $\mathbf{3 . 0 3} \mathbf{B}$ \\
\hline T3=Hand weeding once + inter-row cultivation & 2.62 & 2.56 & 2.52 & $\mathbf{2 . 5 6 ~ C}$ \\
\hline T4=Inter-row cultivation only & 2.39 & 2.39 & 2.35 & $\mathbf{2 . 3 7 ~ D}$ \\
\hline T5=Hand hoeing once & 2.31 & 2.28 & 2.25 & $\mathbf{2 . 2 8 ~ E}$ \\
\hline T6=Hand hoeing twice & 2.23 & 2.17 & 2.13 & $\mathbf{2 . 1 7} \mathbf{~}$ \\
\hline T7=Hand hoeing thrice & 2.11 & 2.05 & 2.00 & $\mathbf{2 . 0 5 ~ G}$ \\
\hline T8=Weedy check & 1.77 & 1.71 & 1.73 & $\mathbf{1 . 7 3 ~ H}$ \\
\hline
\end{tabular}

$\mathrm{SE} \pm=0.0158, \mathrm{LSD} 0.05=0.0339$

\section{Tillers per stool}

The variance analysis showed significant influence $(\mathrm{P}<0.05)$ of treatments on the tillers per stool of sugarcane (Appendix-V). The sugarcane crop attained maximum tillers per stool (7.96) when crop treated with Buctril M @ $3.75 \mathrm{~kg} \mathrm{ha}^{-1}$, closely followed by 7.85 , 7.62 and 7.44 tillers per stool in plots receiving Metribuzin + 2, 4-D @ $3.75 \mathrm{~kg} \mathrm{ha}^{-}$ ${ }^{1}$ on the rows of cane+ inter row cultivation.
Hand weeding once+inter- row cultivation and inter row cultivation only. A simultaneous decline in tillers per stool of the sugarcane crop i.e. $6.28,6.82$ and 6.13 were recorded in plots receiving hand hoeing once, hand hoeing twice and hand hoeing thrice, respectively. However, the shortest tillers per stool on average (2.98) was recorded @ weedy check, where crop treated with weedy check (Table 6).

Table 6. Tillers per stool of sugarcane as influenced by various weed treatment

\begin{tabular}{|c|c|c|c|c|}
\hline Treatment & R I & R II & RIII & Mean \\
\hline TI =Buctril M @ 3.75 $\mathrm{kg} \mathrm{ha}^{-1}$ & 8.00 & 7.92 & 7.97 & $\mathbf{7 . 9 6 ~ A}$ \\
\hline $\begin{array}{c}\text { T2=Metribuzin + 2, 4-D @ 3.75 } \mathrm{kg} \mathrm{ha}^{-1} \text { on cane rows + inter-row } \\
\text { cultivation }\end{array}$ & 7.91 & 7.85 & 7.79 & $\mathbf{7 . 8 5} \mathbf{~ A}$ \\
\hline T3=Hand weeding once + inter-row cultivation & 7.65 & 7.64 & 7.59 & $\mathbf{7 . 6 2 ~ B}$ \\
\hline T4=Inter-row cultivation only & 7.35 & 7.42 & 7.55 & $\mathbf{7 . 4 4} \mathbf{~ C}$ \\
\hline T5=Hand hoeing once & 6.22 & 6.29 & 6.34 & $\mathbf{6 . 2 8 ~ E}$ \\
\hline T6=Hand hoeing twice & 6.92 & 6.82 & 6.73 & $\mathbf{6 . 8 2} \mathbf{~ D}$ \\
\hline T7=Hand hoeing thrice & 6.00 & 6.28 & 6.12 & $\mathbf{6 . 1 3} \mathbf{~}$ \\
\hline T8=Weedy check & 3.01 & 3.00 & 2.95 & $\mathbf{2 . 9 8 ~ G}$ \\
\hline
\end{tabular}

$\mathrm{SE} \pm=0.0682, \mathrm{LSD} 0.05=0.1462$

\section{Weight of 10 canes $(\mathrm{kg})$}

The sugarcane crop attained maximum weight of 10 canes $(18.93 \mathrm{~kg})$ when crop treated with Buctril M @ $3.75 \mathrm{~kg} \mathrm{ha}^{-1}$, closely followed by $18.79 \mathrm{~kg}, 18.65 \mathrm{~kg}$ and $18.03 \mathrm{~kg}$ weight of 10 canes in plots receiving Metribuzin + 2, 4-D @ $3.75 \mathrm{~kg} \mathrm{ha}^{-1}$ on cane rows + inter-row cultivation, Hand weeding once + inter-row cultivation and Inter-row cultivation only. A simultaneous decline in weight of 10 canes of the sugarcane crop i.e. $16.45 \mathrm{~kg}, 15.29 \mathrm{~kg}$ and $14.45 \mathrm{~kg}$ were recorded in plots receiving hand hoeing once, hand hoeing twice and hand hoeing thrice, respectively. However, the shortest weight of 10 canes on average $(6.68 \mathrm{~kg})$ was recorded @ weedy check, where crop treated with weedy check (Table 7). 
Table 7. Weight of 10 canes $(\mathrm{kg})$ of sugarcane as influenced by various weed treatment

\begin{tabular}{|c|c|c|c|c|}
\hline Treatment & R I & RII & R III & Mean \\
\hline $\mathrm{TI}=$ Buctril M @ $3.75 \mathrm{~kg} \mathrm{ha}^{-1}$ & 19.00 & 18.98 & 18.82 & $18.93 \mathrm{~A}$ \\
\hline $\begin{array}{c}\mathrm{T} 2=\text { Metribuzin }+2,4-\mathrm{D} @ 3.75 \mathrm{~kg} \mathrm{ha}^{-1} \text { on cane rows }+ \\
\text { inter-row cultivation }\end{array}$ & 18.76 & 18.83 & 18.79 & 18.79 A \\
\hline T3=Hand weeding once + inter-row cultivation & 18.65 & 18.60 & 18.72 & $18.65 \mathrm{~A}$ \\
\hline T4=Inter-row cultivation only & 18.22 & 18.00 & 17.88 & $18.03 \mathrm{~B}$ \\
\hline T5=Hand hoeing once & 16.60 & 16.42 & 16.33 & $16.45 \mathrm{C}$ \\
\hline T6=Hand hoeing twice & 15.22 & 15.00 & 15.66 & 15.29 D \\
\hline T7=Hand hoeing thrice & 14.30 & 14.45 & 14.62 & $\mathbf{1 4 . 4 5 ~ E}$ \\
\hline T8=Weedy check & 6.80 & 6.52 & 6.73 & $6.68 \mathrm{~F}$ \\
\hline
\end{tabular}

$\mathrm{SE} \pm=0.1379, \mathrm{LSD} 0.05=0.2957$

Cane yield ( $\left(\mathrm{ha}^{-1}\right)$

The analysis of variance exhibited significant $(\mathrm{P}<0.05)$ effect of treatments on the cane yield of sugarcane (Appendix-VII). The sugarcane crop attained maximum cane yield (69.55 $\left.\mathrm{t} \mathrm{ha}^{-1}\right)$ when crop treated with Buctril M @ $3.75 \mathrm{~kg} \mathrm{ha}^{-1}$, closely followed by 67.48 $\mathrm{t} \mathrm{ha}^{-1}, 65.67 \mathrm{t} \mathrm{ha}^{-1}$ and $64.59 \mathrm{t} \mathrm{ha}^{-1}$ cane yield in plots receiving Metribuzin +2, 4-D @ 3.75 $\mathrm{kg} \mathrm{ha}^{-1}$ on cane rows + inter-row cultivation, Hand weeding once + inter-row cultivation and Inter-row cultivation only. A simultaneous decline in cane yield of the sugarcane crop i.e. $63.43 \mathrm{t} \mathrm{ha}^{-1}, 61.68 \mathrm{t} \mathrm{ha}^{-1}$ and $58.13 \mathrm{t} \mathrm{ha}^{-1}$ were recorded in plots receiving hand hoeing once, hand hoeing twice and hand hoeing thrice, respectively. However, the shortest cane yield on average (27.22 $\mathrm{t} \mathrm{ha}^{-1}$ ) was observed @ weedy check, where crop treated with weedy check (Table 8).

Table 8. Cane yield ( $\left.\mathrm{t} \mathrm{ha}^{-1}\right)$ of sugarcane as influenced by various weed treatment

\begin{tabular}{|c|c|c|c|c|}
\hline Treatment & R I & R II & R III & Mean \\
\hline TI =Buctril M @ 3.75 $\mathrm{kg} \mathrm{ha}^{-1}$ & 69.58 & 69.55 & 69.53 & $\mathbf{6 9 . 5 5 ~ A}$ \\
\hline $\begin{array}{c}\text { T2=Metribuzin + 2, 4-D @ 3.75 } \mathrm{kg} \mathrm{ha}^{-1} \text { on cane rows + } \\
\text { inter-row cultivation }\end{array}$ & 67.52 & 67.49 & 67.45 & $\mathbf{6 7 . 4 8 ~ B}$ \\
\hline T3=Hand weeding once + inter-row cultivation & 65.73 & 65.67 & 65.62 & $\mathbf{6 5 . 6 7 ~ C}$ \\
\hline T4=Inter-row cultivation only & 64.62 & 64.59 & 64.56 & $\mathbf{6 4 . 5 9} \mathbf{D}$ \\
\hline T5=Hand hoeing once & 63.32 & 63.44 & 63.54 & $\mathbf{6 3 . 4 3 ~ E}$ \\
\hline T6=Hand hoeing twice & 61.66 & 61.67 & 61.72 & $\mathbf{6 1 . 6 8 ~ F}$ \\
\hline T7=Hand hoeing thrice & 58.15 & 58.12 & 58.13 & $\mathbf{5 8 . 1 3 ~ G}$ \\
\hline T8=Weedy check & 28.24 & 25.23 & 28.21 & $\mathbf{2 7 . 2 2 ~ H}$ \\
\hline
\end{tabular}

$\mathrm{SE} \pm=0.5002, \mathrm{LSD} 0.05=1.0728$

\section{Brix (\%)}

The sugarcane crop attained maximum brix (22.96\%) when crop treated with Buctril M @ $3.75 \mathrm{~kg} \mathrm{ha}^{-1}$, closely followed by $22.75 \%$, $22.37 \%$ and $21.92 \%$ brix in plots receiving Metribuzin + 2, 4-D @ $3.75 \mathrm{~kg} \mathrm{ha}^{-1}$ on the rows of cane+ inter row cultivation. Hand weeding once+inter- row cultivation and inter row cultivation only. A simultaneous decline in brix of the sugarcane crop i.e. $21.36 \%, 21.10 \%$ and $20.33 \%$ were recorded in plots receiving hand hoeing once, hand hoeing twice and hand hoeing thrice, respectively. However, the shortest brix on average (18.94\%) was observed @ weedy check, where crop treated with weedy check (Table 9). 
Table 9. Brix (\%) of sugarcane as influenced by various weed treatment

\begin{tabular}{|c|c|c|c|c|}
\hline Treatment & R I & R II & R III & Mean \\
\hline $\mathrm{TI}=$ Buctril M @ $3.75 \mathrm{~kg} \mathrm{ha}^{-1}$ & 23.00 & 22.91 & 22.97 & $22.96 \mathrm{~A}$ \\
\hline $\begin{array}{c}\mathrm{T} 2=\text { Metribuzin }+2,4-\mathrm{D} @ 3.75 \mathrm{~kg} \mathrm{ha}^{-1} \text { on cane rows }+ \\
\text { inter-row cultivation }\end{array}$ & 22.88 & 22.75 & 22.63 & $22.75 \mathrm{~A}$ \\
\hline T3=Hand weeding once + inter-row cultivation & 22.45 & 22.36 & 22.31 & 22.37 B \\
\hline T4=Inter-row cultivation only & 22.00 & 21.92 & 21.85 & $21.92 \mathrm{C}$ \\
\hline T5=Hand hoeing once & 21.45 & 21.35 & 21.28 & 21.36 D \\
\hline T6=Hand hoeing twice & 21.16 & 21.11 & 21.03 & $21.10 \mathrm{E}$ \\
\hline T7=Hand hoeing thrice & 20.00 & 20.65 & 20.35 & $20.33 \mathrm{~F}$ \\
\hline T8=Weedy check & 18.88 & 19.00 & 18.96 & $18.94 \mathrm{G}$ \\
\hline
\end{tabular}

$\mathrm{SE} \pm=0.1134, \mathrm{LSD} 0.05=0.2433$

\section{Sugar recovery $(\%)$}

The sugarcane crop attained maximum sugar recovery $(11.48 \%)$ when crop treated with Buctril M@ $3.75 \mathrm{~kg} \mathrm{ha}^{-1}$, closely followed by $11.38 \%, 11.19 \%$ and $10.96 \%$ sugar recovery in plots receiving Metribuzin $+2,4$ D@ $3.75 \mathrm{~kg} \mathrm{ha}^{-1}$ on the rows of cane+ inter row cultivation. Hand weeding once+interrow cultivation and inter row cultivation only. A simultaneous decline in sugar recovery of the sugarcane crop i.e. $10.68 \%$, $10.55 \%$ and $10.17 \%$ were recorded in plots receiving hand hoeing once, hand hoeing twice and hand hoeing thrice, respectively. However, the shortest sugar recovery on average $(9.47 \%)$ was observed @ weedy check, where crop treated with weedy check (Table 10).

Table 10. Sugar recovery $(\%)$ of sugarcane as influenced by various weed treatment

\begin{tabular}{|c|c|c|c|c|}
\hline Treatments & R-I & R-II & R-III & Mean \\
\hline T1=Buctril M @ $3.75 \mathrm{~kg} \mathrm{ha}^{-1}$ & 11.50 & 11.46 & 11.49 & $11.48 \mathrm{~A}$ \\
\hline $\begin{array}{r}\mathrm{T} 2=\text { Metribuzin }+2,4-\mathrm{D} @ 3.75 \mathrm{~kg} \mathrm{ha}^{-1} \text { on cane rows }+ \text { inter- } \\
\text { row cultivation }\end{array}$ & 11.44 & 11.38 & 11.32 & $11.38 \mathrm{~A}$ \\
\hline T3=Hand weeding once + inter-row cultivation & 11.23 & 11.18 & 11.16 & $11.19 \mathrm{~B}$ \\
\hline T4=Inter-row cultivation only & 11.00 & 10.96 & 10.93 & $10.96 \mathrm{C}$ \\
\hline T5=Hand hoeing once & 10.73 & 10.68 & 10.64 & $10.68 \mathrm{D}$ \\
\hline T6=Hand hoeing twice & 10.58 & 10.56 & 10.52 & $10.55 \mathrm{E}$ \\
\hline $\mathrm{T} 7=$ Hand hoeing thrice & 10.00 & 10.33 & 10.18 & $10.17 \mathrm{~F}$ \\
\hline T8=Weedy check & 9.44 & 9.50 & 9.48 & $9.47 \mathrm{G}$ \\
\hline
\end{tabular}

$\mathrm{SE} \pm=0.0572, \mathrm{LSD} 0.05=0.1226$

\section{Discussion}

Present investigation was performed at Agriculture Research Institute, Sugarcane Section, Tandojam with 3 replications RCBD in with $(8 \mathrm{~m} \times 3 \mathrm{~m}) 24 \mathrm{~m}^{2}$ size of plot. This investigation was performed on the plot of four weed species were recorded which included Chaff-flower, Green amaranth, Creeping thistle and Lamb's quarters. The above record of the weed flora was maintained sowing of sugarcane. The sugarcane crop @ Buctril M @ $3.75 \mathrm{~kg} \mathrm{ha}^{-1}$ resulted in $30.76 \mathrm{~m}^{-2}$ weed density, $80.20 \%$ weed reduction, 7.96 tillers stool ${ }^{-1}, 265.33$ $\mathrm{cm}$ length of cane, $69.55 \mathrm{t} \mathrm{h}^{-1} 3.08 \mathrm{~cm}$ girth of cane, $18.93 \mathrm{~kg}$ weight of ten canes. $22.95 \%$ brix and $11.48 \%$ sugar recovery. In same way the crop that were treated with (Metribuzin+2,4D @ 3.75 $\mathrm{kg} \mathrm{h}^{-1}$ the rows of cane + cultivation of inter- row in the result of $36.70 \mathrm{~m}^{-2}$ density, $76.37 \%$ reduction of weed, $256.33 \mathrm{~cm}$ length of cane with 3.30 girth, 7.85 stool $^{-1}$ tillers, $67.48 \mathrm{t} \mathrm{ha}^{-1}$ yield of cane, $18.79 \mathrm{~kg}$ weight of 10 canes, $11.38 \%$ 
sugar recovery and $22.75 \%$ brix. The sugarcane crop which were treated once with hand weeding + inter row cultivation hence resulting $37.81 \mathrm{~m}^{-2}$ density of weed with $75.68 \%$ reduction, $2.56 \mathrm{~cm}$ length of cane, $65.67 \mathrm{t} \mathrm{ha}^{-1}$ brix. The sugarcane crop which were treated with inter row crop cultivation produced only, $44.16 \mathrm{~m}^{-2}$ weed density, $71.59 \%$ weed reduction, length of cane $247.00 \mathrm{~cm}, 7.44$ tillers stool ${ }^{-1} 2.37 \mathrm{~cm}$ girth of cane, $18.03 \mathrm{~kg}$ weight of 10 canes, yield of cane $64.59 \mathrm{t} \mathrm{h}^{-1} 21.92 \%$ brix and sugar recovery $10.96 \%$. The sugarcane crop treated with handing once result of $53.94 \mathrm{~m}^{-2}$ reduction of weed $242.67 \mathrm{~cm}$ length of cane, $65.29 \%$ weed density, 6.2844 tillers stool $^{-1}$, weight of 10 canes $16.45 \mathrm{~kg}$, yield of cane $63.43 \mathrm{ha}^{-1} 21.36 \%$ brix and $10.68 \%$ recovery of sugar. The crop treated under handing twice resulted in $71.52 \mathrm{~m}^{-2}$ weed density, $53.96 \%$ weed reduction, Length of cane 253.67, 6.82 tillers stool $^{-1}, 2.17 \mathrm{~cm}$ girth of cane, weight of 10 canes $15.29 \mathrm{~kg}$ yield of cane $61.68 \mathrm{t} \mathrm{ha}^{-1}$ brix $21.10 \%$ and sugar recovery $10.55 \%$. The sugar cane crop that were treated with hand 3 time result showed $94.27 \mathrm{~m}^{-2}$ weed density, weed reduction $39.33 \%$, yield of cane $192.00 \mathrm{~cm}$, girth 2.05 $\mathrm{cm}$, tillers stool ${ }^{-1} 6.13$, weight of 10 canes $14.45 \mathrm{~kg}$ cane yield $58.13 \mathrm{t} \mathrm{ha}^{-1}$ brix 20.33 and recovery sugar $10.17 \%$. The sugarcane crop that were treated with check weedy were produced $155.45 \mathrm{~m}^{2}, 0.00 \%$ density of weed reduction 161.67 , length of cane $1.73 \mathrm{~cm}$, cane girth, tillers stollol ${ }^{-1} 6.68 \mathrm{~kg}$ weight of 10 canes, 18.94 brix, $27.22 \mathrm{t} \mathrm{ha}^{-1}$, sugar recovery $9.47 \%$. It was concluded that the crop treated with Buctril M @ $3.75 \mathrm{~kg} \mathrm{ha}^{-1}$ resulted in highest cane yield $\left(69.55 \mathrm{t} \mathrm{ha}^{-1}\right)$. Sugarcane is the most important sugar crop in Pakistan occupying an area 1217 thousand hectares with an average productivity 65.5 million tons (Govt. of Pakistan 2017). This low productivity is mainly due to heavy weed infestation in early growth stage and poor weed management practices [8]. Initial slow growth and wider row spacing provide ample opportunity for weeds to occupy the vacant spaces between rows and offer serious cropweed competition. [9] Reported yield loss to an extent of $28-38 \%$ in ratoon crop due to weeds and the much critical period for competition of weed among 30 to 60 days after the ratooning. Besides this, manual hand weeding is not much effective against perennials weeds like Cyperus rotundas particularly in sugarcane ratoon [10]. Moreover, timely availability of agricultural labor is a problem and manual weeding is laborious and costly in cultural method of weed control. On the other hand, chemical method of weed control not only save agricultural labour, time but also effectively control weeds. It was concluded that the crop treated with Buctril M @ 3.75 kg ha ${ }^{-1}$ resulted in highest cane yield $\left(69.55 \mathrm{t} \mathrm{ha}^{-1}\right)$. Mention results are in support with the other investigation of various investigators [11], reported that overall population of weed varies from crop to crop depending on weed emergence time, weed type, environmental factors and management practices Ibrahim [12-15].

\section{Conclusions}

It was concluded that the crop treated with Buctril M @ $3.75 \mathrm{~kg} \mathrm{ha}^{-1}$ resulted in highest cane yield (69.55 $\left.\mathrm{t} \mathrm{ha}^{-1}\right)$.

\section{Authors' contributions}

Conceived and designed the experiments: Q Jogi, Performed the experiments: MM Kandhro, AN Shah \& GA Hajano, Analyzed the data: AH Soomro, ZA Abbasi \& M Buriro, Contributed reagents/ materials/ analysis tools: Q Jogi \& ZA Kalwar, Wrote the paper: AJ Soomro \& ZA Kalwar.

\section{References}

1. Mahar MT, Khuhawar MA, Baloch MA \& Jahangir TM (2012). Effects of spent wash of ethanol industry on groundwater: A case study of Rahimyar Khan District, Pakistan. J of Environ Sci and Water Res (4): 85-94. 
2. Ansari F, Awasthi AK \& Srivastava BP (2012). Physico-chemical characterization of distillery effluent and its dilution effect at different levels. Arch of App Sci Res 4(4): 1705-715.

3. GoP (2017). Sugarcane: Economic survey of Pakistan 2016-2017. Department of Food and Agriculture, Bureau of Statistics (Economic Wing), Government of Pakistan, Islamabad.

4. Panda SC (2006). Crop management and integrated farming: Systems of crop production. Agrobios (India): pp 53-73.

5. Taye E (1991). Survey of weed flora and evaluation of some foliage-applied herbicides in the sugarcane plantation of Wonji-Shoa and Methara. MSc. thesis. Alemaya University of Agriculture, Alemaya, Ethiopia.

6. Ballaré CL \& Casal JJ (2000). Light signals perceived by crop and weed plants. J Crop Res 67(1): 149-160.

7. Statistix (2006). Statistix 8 users guides version 1.0. Analytical software, PO box 12185, tallahasee FI 32317 USA. Copyright (C) 2006 by analytical software.

8. Singh D \& Tomar PK (2015). Productivity of sugarcane ratoon influenced by weed management practices. Ind J Sci 55(1): 25-29.
9. Srivastava TK, Singh AK \& Srivastava SN (2012). Critical period of crop-weed competition in sugarcane ratoon. Indian $J$ Weed Sci 34(4): 320-321.

10. Mishra A, Mishra AK, Ghosh AK \& Jha S (2012). Standardization of a traditional polyherbo-mineral formulation brahmi vati. J of Agri Sci 10(3): 390-396.

11. Ibrahim AAS (2013). Efficiency of some herbicides for weed control in sugarcane. J of Pla Sci 21(3): 255-258.

12. Khan T, Faheem A \& Muhammad Z (2015). Weeds and weed control methods in sugarcane: a case study of Khyber Pakhtunkhwa Pakistan. Pak J of Weed Sci Res 21(2): 217-228.

13. Suwanarak K (2012). Weed management in sugar cane in Thailand. Symposium on weed management held in Bogor, Indonesia, 7-9 June 1989. Biotropspecial publication 38: 199-214.

14. Tahir M \& Ismail M (2016). Area, production and yield of sugarcane in major countries. Website of Sugar Crops Research Institute, Mardan (KPK) last updated on April 10, 2016. www.scri.gkp.pk.

15. Wilson EJJ, Griffin L, Jones CA \& Etheredge LM (2017). Weed Control and Economics Using Reduced Tillage Programs in Sugarcane. J of Pla Sci 20(2): 319-325. 